\title{
MATHEMATICAL MODELING OF ARGON AND NITROGEN PLASMA ARCS AT ATMOSPHERIC PRESSURE*
}

José Alfredo Delgado Álvarez'

Patricio Fernando Mendez ${ }^{2}$ Marco Aurelio Ramírez-Argáez $z^{3}$

\begin{abstract}
A 2D mathematical model was developed for the plasma arc welding process. Computational simulations based on mass and momentum conservation equations as well as Maxwell equations were performed by using the commercial software PHOENICS. The model predicts the electric characteristics of the arc column, flow patterns, temperature contours and electrical potential, by varying the composition of the cover gas, in this case, argon and nitrogen. It was found that an arc of nitrogen with respect to one of argon generates more intense jets, greater voltages, and lower temperatures in the arc column for welding arcs current of $150 \mathrm{~A}$ and $10 \mathrm{~mm}$ arc length.

Keywords: Electric arc, heat transfer, fluid flow, mathematical modeling.

1 Ph.D. student, Universidad Nacional Autónoma de México/Metallurgical Engineering Department, Facultad de Química, Mexico city, Mexico.

2 Professor, Universidad Nacional Autónoma de México/Metallurgical Engineering Department, Facultad de Química, Mexico city, Mexico.

3 Professor, Alberta University/Department of Chemical and Materials Engineering, Edmonton, Canada.
\end{abstract}




\section{INTRODUCTION}

Within the alternative manufacturing processes, welding is one of the fastest, efficient and economical methods for joining metals, and at the industrial scale is of enormous importance especially in sectors such as the metalworking industry, auto parts and construction. From all available welding processes, electric arc welding excels in the applications in which it can be used. The understanding of the magnetohydrodynamic phenomena (MHD) of the system is fundamental and represents the only way to be able to optimize diverse process variables to obtain an optimum performance in the welding process.

There are numerous and diverse mathematical modeling works on arc welding processes. The first attempt to describe the physics involved in electric arcs was made by Maecker [1] who was the first to explain the generation of high-intensity jet produced by electromagnetic forces at high currents and derived expressions for maximum velocity and pressure within the arc through Maxwell's equations. Research by Lowke [2], Squire [3], Ramakrishnan [4,5], and Allum [6] derived analytical expressions to relate the characteristics of the arc such as voltage, electric field, arc radius and velocity plasma as a function of the arc length, the arc current, the arc temperature and the gas pressure. However, these analytic expressions were obtained by neglecting the viscous effects and assuming isothermal arcs, constant physical properties and a simple arc shape.

As computing capacities increase, numerical solutions to realistically describe a power arc become a very powerful tool. Some efforts to model an electric arc in welding processes were performed by Hsu et. al. [7], Mckelliget and Szekely [8], Choo et. al. [9] and Kim et. al. [10] to mention a few. These works simulated argon arcs as cover gas, using a current density at one point of the cathode, $J_{c}$, as the only adjustable parameter. These works reproduce temperature fields, current and heat flux densities at the surface of the anode and all of them are in good agreement with experimental measurements [7, 11].

Currently the mathematical models of electric arc welding deal with problems such as weld pool depression [12,13], the influence of electrode geometry on the electric characteristics of the arc $[14,15]$, phenomena in the Interfaces of the electrodes and the arc [16,17], and the formation of droplets [18]. This work aims to analyze how the electric properties of the arc are affected by varying the composition of the cover gas, for which are presented simulations at steady state of electric arcs burning in atmospheres of argon and nitrogen.

\section{MATHEMATICAL MODELLING}

\subsection{Assumptions}

The model contains the following simplifying assumptions:

- Magnetic convection is negligible compared with magnetic diffusion. This is a result of the small magnetic Reynolds number, which represents the ratio of magnetic convection to magnetic diffusion, and is defined as

$$
R_{e, m}=V_{0} L_{0} \sigma \mu_{0}
$$


where $V_{0}$ and $L_{0}$ are the characteristic velocity and length scale of the welding process respectively, $\sigma$ and $\mu_{0}$ are the electric conductivity and magnetic permeability, respectively.

- The plasma is in LTE. This implies that electrons and heavy particle temperatures are the same. This assumption is known to be valid in the arc column region but will break down near the electrodes [19].

- Physical properties are considered to be a function of only the gas temperature.

- The problem is set-up using axisymmetric two-dimensional cylindrical polar coordinates.

- The plasma is optically thin, i.e. no absorption is taking place inside the arc column.

- The system is assumed to be at steady state since a DC system is modeled.

- The anode surface is considered to be flat. This is certainly an oversimplification since weld pool depression is a very well documented issue [10].

- At the cold electrode surfaces, the electrical conductivity corresponds to that of the higher temperature electrons.

- The flow is assumed to be laminar. The assumption of laminar flow can be justified by taking characteristic values of the parameters in a welding arc process to compute the Reynolds number. Reynolds numbers ranging from 500 to 2000 are common in this process while, in a free jet, the transition from the laminar to turbulent flow regime is found to take place at Reynolds numbers around 100,000.

- The current density is assumed to be uniform across a cathode spot of specified radius, and zero outside the cathode spot. A current density of $6.5 \times 10^{7} \mathrm{Am}^{-2}$ is assumed. This value is assumed to be independent of arc length and arc current, although it will depend on cathode material [8].

\subsection{Governing equations}

The governing equations are:

Conservation of mass:

$$
\frac{\partial}{\partial z}\left(\rho v_{z}\right)+\frac{1}{r} \frac{\partial}{\partial r}\left(\rho r v_{r}\right)=0
$$

Conservation of axial momentum:

$$
\frac{\partial}{\partial z}\left(\rho v_{z}^{2}\right)+\frac{1}{r} \frac{\partial}{\partial r}\left(\rho r v_{r} v_{z}\right)=-\frac{\partial P}{\partial z}+\frac{2 \partial}{\partial z}\left(\mu \frac{\partial v_{z}}{\partial z}\right)+\frac{1}{r} \frac{\partial}{\partial r}\left(r \mu\left(\frac{\partial v_{z}}{\partial r}+\frac{\partial v_{r}}{\partial z}\right)\right)-B_{\Theta} J_{r}
$$

Conservation of radial momentum:

$$
\frac{\partial}{\partial z}\left(\rho v_{r} v_{z}\right)+\frac{1}{r} \frac{\partial}{\partial r}\left(\rho r v_{r}^{2}\right)=-\frac{\partial P}{\partial r}+\frac{\partial}{\partial z}\left\{\mu\left(\frac{\partial v_{r}}{\partial z}+\frac{\partial v_{z}}{\partial r}\right)\right\}+\frac{2}{r} \frac{\partial}{\partial r}\left(\mu \frac{r \partial v_{r}}{\partial r}\right)-\frac{2 v_{r}}{r^{2}} \mu+B_{\Theta} J_{r}
$$


where $v_{r}$ is the radial velocity component, $v_{z}$ is the axial velocity component, $P$ is the pressure, $\rho$ is the density, $\mu$ is the molecular viscosity, $J_{r}$ is the radial current density, $J_{z}$ is the axial current density, and $B_{\Theta}$ is the azimuthal magnetic flux density vector.

Conservation of energy:

$$
\frac{\partial}{\partial z}\left(\rho v_{z} h\right)+\frac{1}{r} \frac{\partial}{\partial r}\left(\rho r v_{r} h\right)=\frac{\partial}{\partial z}\left\{\frac{k}{C_{p}} \frac{\partial h}{\partial z}\right\}+\frac{1}{r} \frac{\partial}{\partial r}\left\{\frac{k r}{C_{p}} \frac{\partial h}{\partial r}\right\}+S_{t}
$$

The source term $S_{t}$ is represented by

$$
S_{t}=\left(\frac{J_{z}^{2}+J_{r}^{2}}{\sigma}\right)-S_{r}+\frac{5}{2} \frac{k_{B}}{e}\left\{\frac{J_{z}}{C_{p}} \frac{\partial h}{\partial z}+\frac{J_{r}}{C_{p}} \frac{\partial h}{\partial r}\right\}
$$

where $h$ is the enthalpy, $C_{p}$ is the specific heat, $S_{r}$ is the radiation losses per unit volume, $k_{B}$ is Boltzmann's constant, and $e$ is the electronic charge. Three source terms from left to right in equation (6) are the Joule heating, the radiation losses and the Thompson effect.

Maxwell equations:

$$
\begin{aligned}
\nabla \times \vec{E} & =0 \\
\nabla \times \vec{H} & =\vec{J} \\
\nabla \cdot \vec{B} & =0
\end{aligned}
$$

Charge conservation equation:

$$
\nabla \cdot \vec{J}=0
$$

Ohm's law:

$$
\vec{J}=\sigma \vec{E}
$$

where $\vec{E}$ is the electric field vector, $\vec{J}$ is the current density vector, $\vec{H}$ is the magnetic field vector, and $\vec{B}$ is the magnetic flux density vector.

Combining equations (7)-(11) two possible formulations of the electromagnetic field can be derived [20], of which we only use the formulation for the electrical potential in this work.

\subsection{Formulation of electrical potential}

Combining the charge conservation equation (equation (10)) and Ohm's law (equation (11)), the electric potential equation is derived as:

$$
\frac{1}{r} \frac{\partial}{\partial r}\left(\sigma \frac{\partial \Phi}{\partial r}\right)+\frac{\partial}{\partial z}\left(\sigma \frac{\partial \Phi}{\partial z}\right)=0
$$

The electric potential, $\Phi$, is defined as 


$$
\vec{E}=-\nabla \Phi
$$

After solving equation (11) it is possible to obtain both current density components using equations (10) and (12):

$$
\begin{gathered}
J_{r}=-\sigma \frac{\partial \Phi}{\partial r} \\
J_{z}=-\sigma \frac{\partial \Phi}{\partial z}
\end{gathered}
$$

Finally, assuming that most of the current can be represented by the axial component, the B field can be derived from Ampere's law (equation (8)):

$$
B=\frac{\mu_{0}}{r} \int_{0}^{r} J_{z} r d r
$$

In this way, the magnetic problem is completely solved. To summarize, the electric potential approach solves two components of the Navier-Stokes equations, the energy conservation equation, the continuity, and the electrical potential equations. Equations (14)-(16) are used to obtain the rest of the electric information that is needed for the sources in the momentum and energy conservation equations.

\subsection{Boundary conditions}

Figure 1 shows a scheme of the computational domain where the boundary conditions are presented.

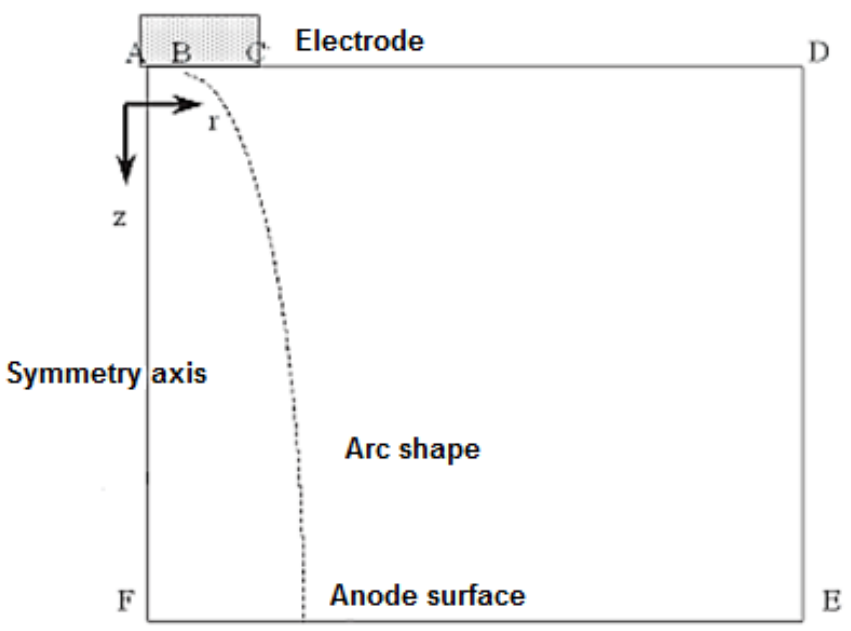

Figure 1. Computation domain.

The most important boundary conditions are those for $\Phi$ y $B$ at the cathode spot. The current density of the cathode spot, $J_{c}$, with a constant value of $6.5 \times 10^{7} \mathrm{Am}^{-2}$, was used as a flux for $\Phi$ and was also used in Ampere's law to get $B$ in the cathode spot. At the anode and cathode surfaces, zero velocities are set. At the symmetry axis, zero gradients are assumed for all variables except $B$ and radial velocity, which are 
both equal to zero. For enthalpy, fixed values were used as boundary conditions at the cathode $\left(7.2 \times 10^{6} \mathrm{Jkg}^{-1}\right.$ corresponding to $\left.4000 \mathrm{~K}\right)$ and at the anode $\left(5.2 \times 10^{5} \mathrm{Jkg}^{-1}\right.$ corresponding to $1000 \mathrm{~K}$ ). However, special attention must be devoted to the specification of the heat fluxes at the anode and cathode surfaces.

At the cathode besides setting the value of the enthalpy at the surface, the presence of a voltage drop (known as cathode fall) is associated with a heat flux expressed by:

$$
Q_{c}=\left|J_{c}\right| V_{c}
$$

where $V_{c}$ is the value of the voltage drop at the cathode in volts. This is a positive source of energy in the energy conservation equation that heats up the arc.

At the anode. Several mechanisms of heat transport need to be considered at the anode for a realistic representation of the heat flux from the arc. This is especially important in coupling the arc with the bath region. A detailed description of each mechanism of heat transfer is given below:

Anode fall: It is the voltage drop that is present at the anode. Electrons passing this voltage drop liberate energy in the process. The energy released due to anode fall is expressed in an analogous manner to equation (17),

$$
Q_{a}=\left|J_{a}\right| V_{a}
$$

here $J_{a}$ is the current at the anode, and $V_{a}$ is the anode voltage drop in volts. Convective heat: A high-velocity jet impinging on the anode surface heats the working piece by convective energy transport. Convective heating is calculated assuming that the rate of heat transfer is governed by an empirical correlation obtained from studies of jets impinging on solid surfaces [8]:

$$
Q_{\text {conv }}=\frac{0.915}{\lambda_{w}}\left[\left(\frac{\rho_{b} \mu_{b}}{\rho_{w} \mu_{w}}\right)^{0.43}\right]\left[\rho_{w} \mu_{w} \frac{d v_{r}}{d r}\right]\left[h_{b}-h_{w}\right]
$$

where $\lambda_{w}$ is the Prandtl number, and subscript $w$ denotes property values at the surface of the working piece while subscript $b$ denotes property values at the edge of the boundary layer. Bath enthalpy, $h_{w}$, is taken to correspond to a temperature of $1000 \mathrm{~K}$.

Radiative heat transfer: Radiative heat transfer from the arc to the anode surface is calculated by means of approximate view factors described by the following equation:

$$
Q_{r a d, i}=\int \frac{S_{r}}{4 \pi r_{i, j}^{2}} \cos \Psi d V_{j}
$$

where $S_{r}$ represents radiation losses per unit volume, $r_{i, j}$ is the vector joining each surface element at the anode to each volume element in the arc, $V_{j}$, and $\Psi$ is the angle between $r_{i, j}$ and the vector normal to the anode surface.

Thompson effect: Transport of thermal energy by hot electrons is called the Thompson effect, which can be described by the equation 


$$
Q_{e}=\frac{5 J_{a}}{2 e} k_{b}\left(\alpha T_{b}-T_{w}\right)
$$

The parameter $\alpha$ represents the ratio of electrons to plasma temperature at the anode fall (at the anode fall the assumption of LTE is no longer valid). In our calculations, $\alpha$ was assumed to have a value of 1.2.

Condensation of electrons: Heat produced due to the condensation of electrons entering from the plasma phase to the liquid metal, can be described by

$$
Q_{\text {cond }}=J_{a} \Phi_{w}
$$

where $\Phi_{w}$ is the work function of the melt pool. For steel, the work function plus the anode fall $\left(\Phi_{w}+V_{a}\right)$ is equal to $6.76 \mathrm{~V}[8]$.

Total anode heat flux: Therefore, the total contribution of heat flux from the arc to the anode may be expressed by the following equation:

$$
Q_{\text {total }}=Q_{\text {rad }}+Q_{\text {conv }}+Q_{\text {cond }}+Q_{e}+Q_{a}
$$

\subsection{Physical properties}

Physical properties were obtained from the data of Boulos et al [19], who reported an extensive list of physical properties calculated for argon as a function of temperature. However, these properties are limited to temperatures up to $25000 \mathrm{~K}$.

\section{SOLUTION TECHNIQUE}

The mathematical model presented above was solved using the control volume technique proposed by Patankar [21] and implemented in the commercial fluid dynamics software PHOENICS. FORTRAN subroutines were written to handle the magnetic problem, special sources appearing in each equation, as well as some special boundary conditions. These subroutines were linked to the code to solve the welding arc problem.

Converged solutions were obtained when conservation imbalances in the domain were below $1 \%$ for all conservation equations. The number of iterations required to achieve convergence varied according to the grid used, and also according to the specific conditions of the calculations.

\section{RESULTS AND DISCUSSION}

\subsection{Velocity vectors fields}

Figure 2 show velocity vector maps for electric arcs operating under the same conditions of the arc with a length of $1 \mathrm{~cm}$ and $150 \mathrm{~A}$ of arc current but being covered by different gas atmospheres (a) argon and (b) nitrogen. Both cases show welldefined jets going from the cathode to the weld pool. These jets are driven by electromagnetic body forces.

By comparing the jets created under these different gas atmospheres, it can be seen that the argon jet is more expanded than the nitrogen jet, which may be due to a lower electric conductivity of the nitrogen plasma in comparison with the argon plasma and then, the electric flow is restricted to a lower arc transversal area for the 
nitrogen arc. This small transversal section area in the nitrogen arc brings other phenomena associated, such as the increment in the current density at the axis of symmetry, which in turn increases the Lorentz's forces (given by the cross product $\mathrm{JxB}$ ) and consequently the resulting velocities in the nitrogen jet are higher than the velocities in the argon jet. Maximum velocities computed are $200 \mathrm{~m} / \mathrm{s}$ and $620 \mathrm{~m} / \mathrm{s}$ for argon and nitrogen arcs respectively.
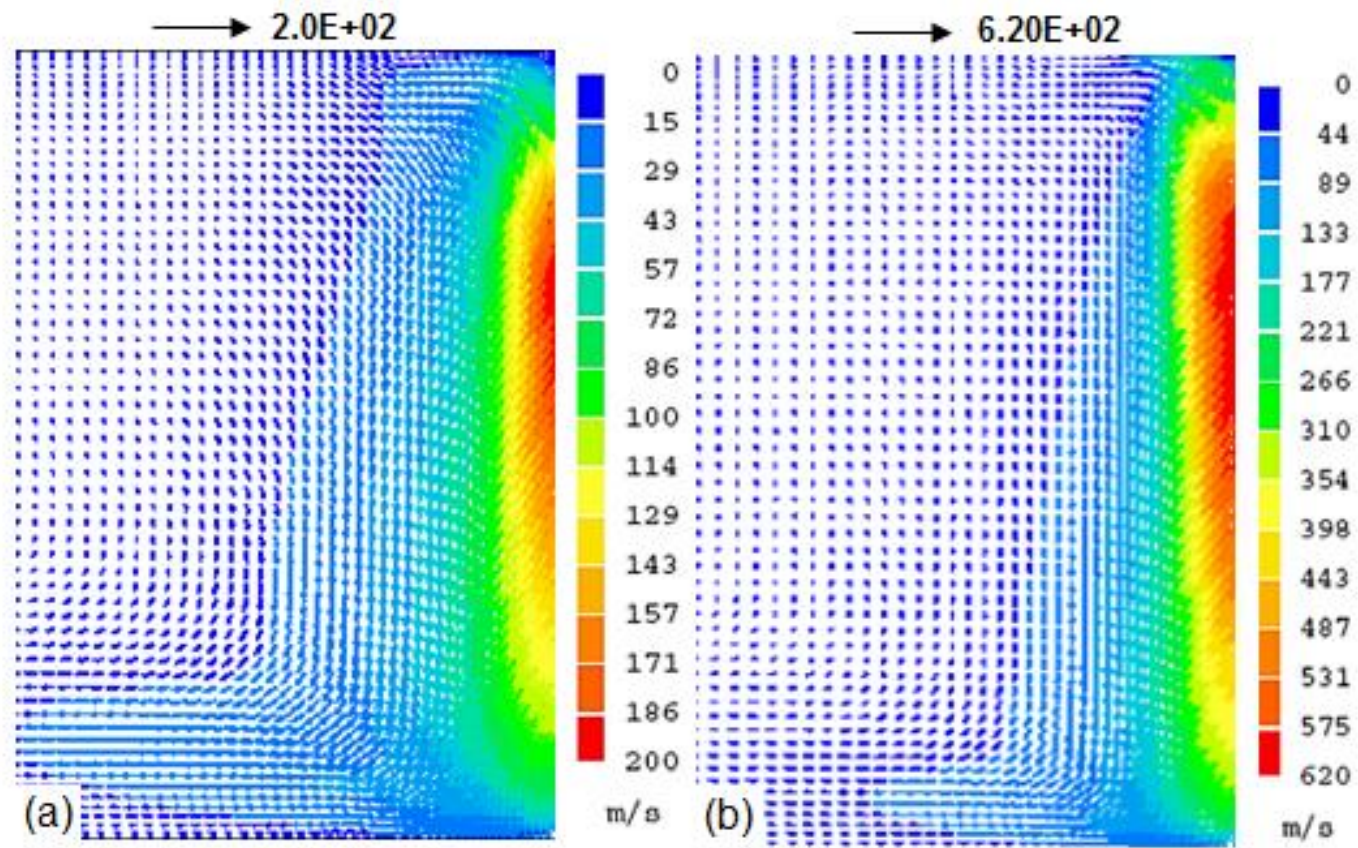

Figure 2. Velocity vector fields for electrics arcs of $150 \mathrm{~A}$ current and $0.1 \mathrm{~m}$ length for (a) argon and (b) nitrogen.

\subsection{Temperature contours}
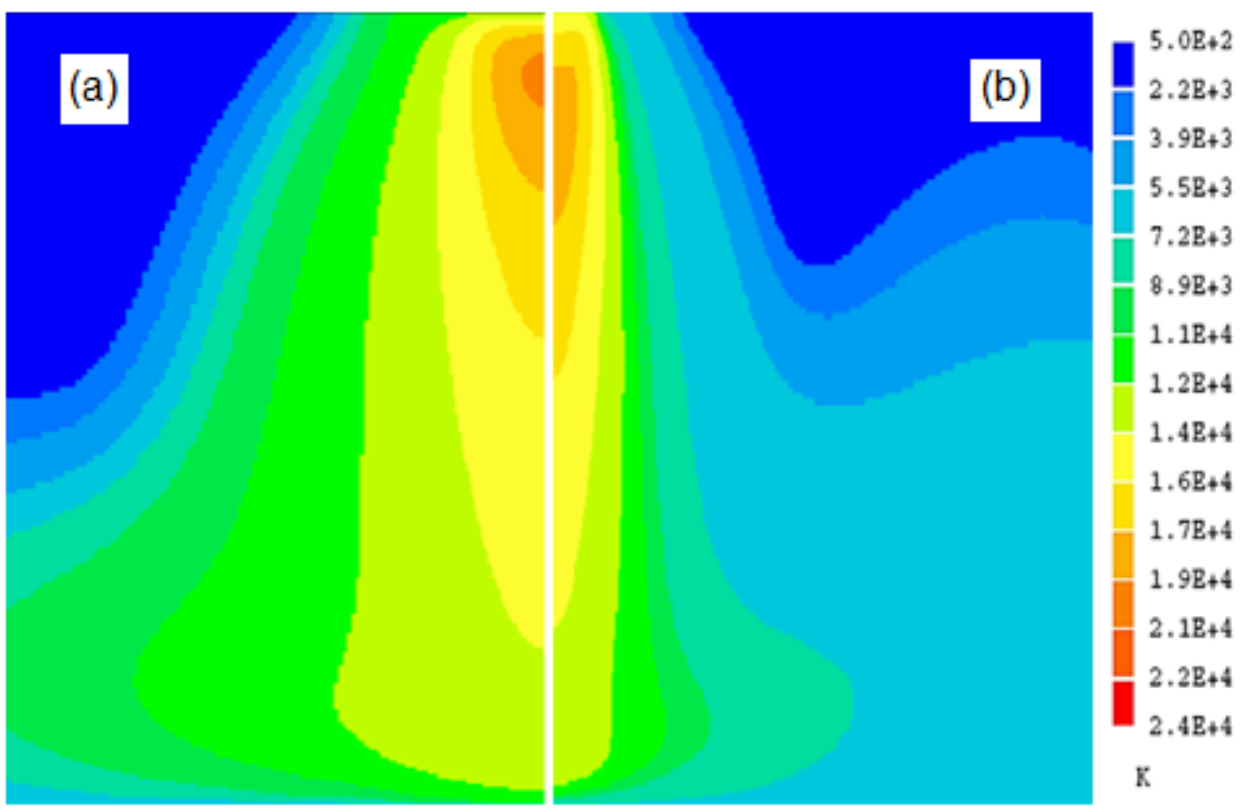

Figure 3. Temperature contours for electrics arcs of $150 \mathrm{~A}$ current and $0.1 \mathrm{~m}$ length for (a) argon and (b) nitrogen. 
Figure 3 show temperature contours for argon (a) and nitrogen (b) arcs with the same arc current and length of $150 \mathrm{~A}$ and $0.1 \mathrm{~m}$ respectively. Temperature contours in the arc column are shaped by the Joule heating effect (the Joule effect depends on the electric conductivity and the arc current density), by the convection from the jet, by the radiation losses, by the Thompson effect and by the specific heat of the shielding gas. The more expanded arc of argon is clearly seen in the temperature contour plot of Figure 3a in comparison with the less expanded arc of nitrogen seen in Figure $3 b$. This finding is in agreement with the discussion given above for the formation of the jet, which involves a lower electric conductivity of nitrogen than the electric conductivity of argon. Other difference in the temperature contour plots is that the maximum temperature of the argon arc is around $24,000 \mathrm{~K}$ while the maximum temperature in the nitrogen arc is around $22,000 \mathrm{~K}$. Of course, the low electric conduction area in the nitrogen arc results in greater current fluxes and more Joule heating, consequently one would expect a hotter nitrogen plasma for nitrogen than for the argon arc. However, the extremely high heat convection in the nitrogen jet dissipates the Joule heating much better in this nitrogen arc than the moderate convection present in the argon arc that can't dissipate the heat in the arc and consequently the argon arc is hotter near the cathode than the nitrogen arc.

\subsection{Voltage contours}

Figure 4 show voltage contours for the arcs of (a) argon and (b) nitrogen under the already mentioned arc conditions. The total voltage drop of the arc under the same current conditions is due to the total ohmic resistance of the plasma, which in turn depends on the electric conductivity and on the arc length. Since the arc length is the same for both cases (Figure 4), the ohmic resistance defines the voltage drop. In this sense, the nitrogen arc has a large voltage drop of $34 \mathrm{~V}$ against the $16.1 \mathrm{~V}$ of voltage drop for the argon arc. This result confirms that the average electric conductivity of nitrogen is lower than that of argon.
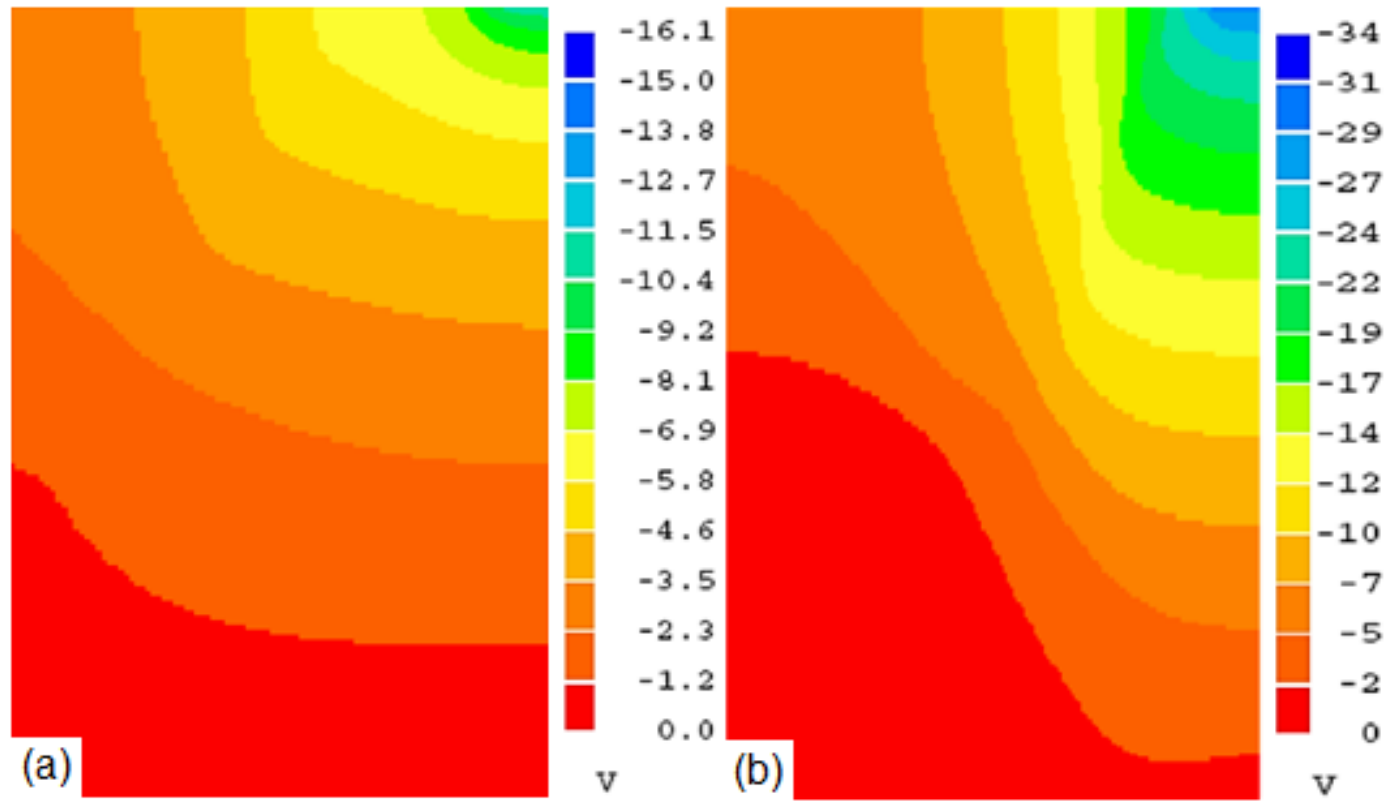

Figure 4. Voltage contours for electrics arcs of $150 \mathrm{~A}$ current and $0.1 \mathrm{~m}$ length with (a) argon and (b) nitrogen.

\subsection{Heat Flows}


Finally, Figs. 5-6 show the total radial heat flux profiles at the anode surface as well as the individual contribution to the total heat flux for each heat transfer mechanism from the arc to the weld pool present in the welding process (convection, radiation, Thompson effect and work function). The heat fluxes for argon plasma and nitrogen plasma are plotted in Fig. 5 and Fig. 6 respectively. The most important heat transfer mechanisms in both cases are the work function and the convection from the plasma jet.

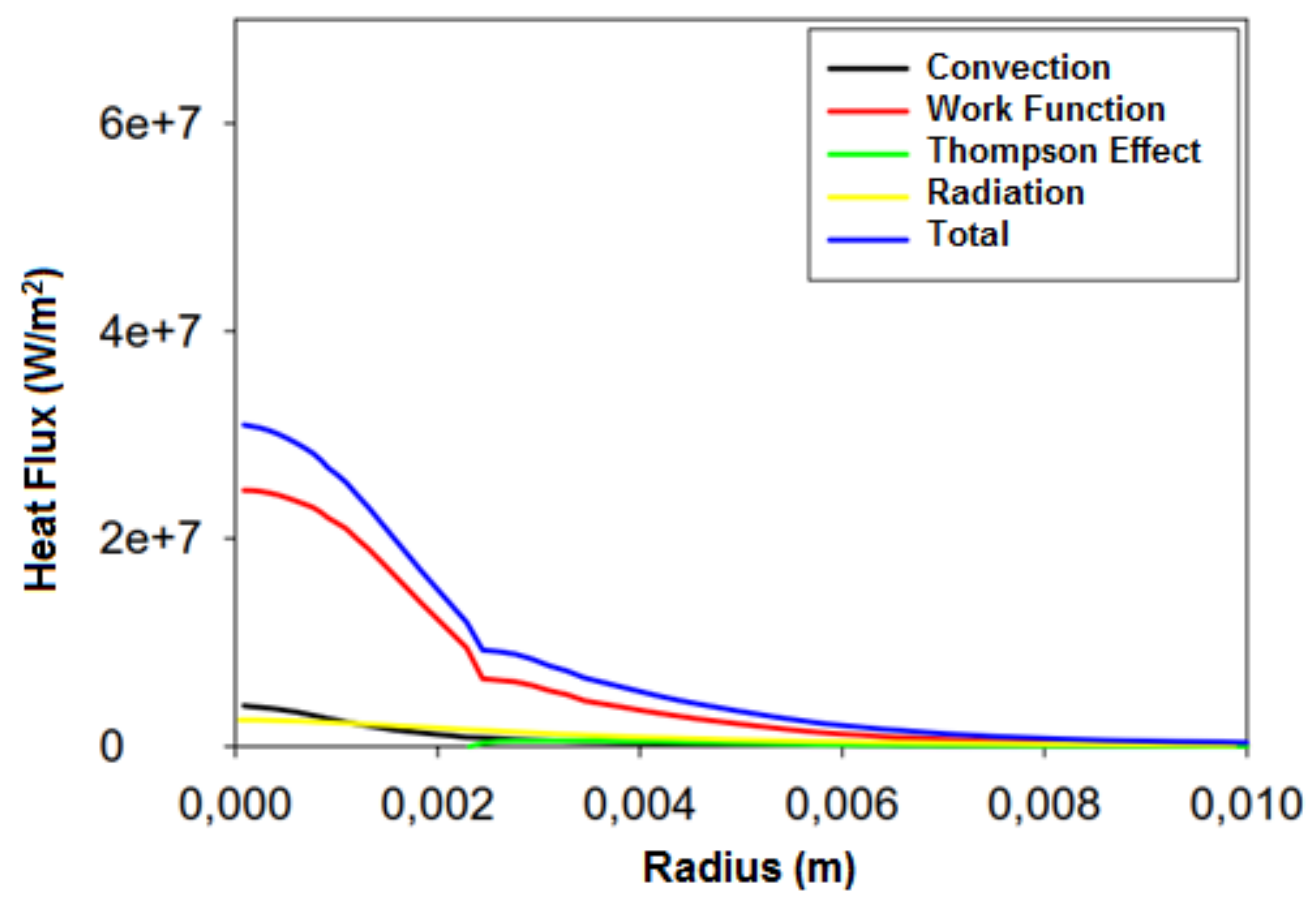

Figure 5. Anode heat flux distribution for an electric arc of $150 \mathrm{~A}$ current and $0.1 \mathrm{~m}$ length with argon.

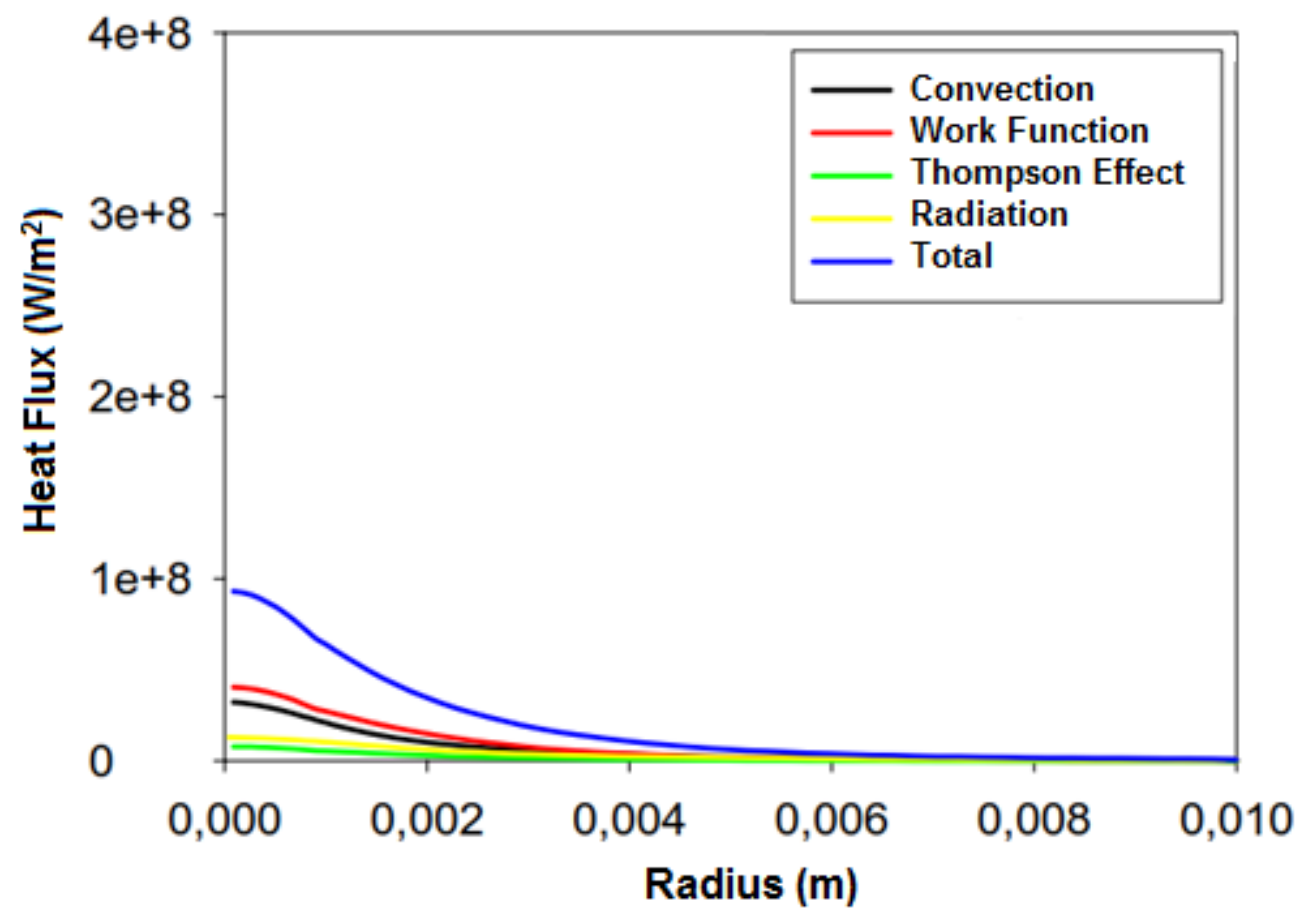


Figure 6. Anode heat flux distribution for an electric arc of $150 \mathrm{~A}$ current and $0.1 \mathrm{~m}$ length with nitrogen.

It is clearly observed that the nitrogen arc transfers more heat near the center than the argon arc. This is due to a narrower arc in the case of nitrogen than in the case of argon. However, as the radial distance increases, both arcs transfer similar heat fluxes and eventually at radial distances above $5 \mathrm{~mm}$ the nitrogen arc does not supply any more heat to the anode while the argon arc still transfers heat beyond this distance and up to a distance of $8 \mathrm{~mm}$. In summary, the nitrogen arc focuses the heat transferred to the anode in a radius of approximately $4 \mathrm{~mm}$ of the weld pool with large heat fluxes, while the argon arc spreads more the heat flux to a circular area of the weld pool of almost $1 \mathrm{~cm}$ in radius but with lower heat fluxes.

\section{CONCLUSIONS}

- An atmosphere of nitrogen promotes lower temperatures, but higher velocities in the plasma jet and voltages within the arc, being several physical properties of gases fundamental to this, such as the electric conductivity.

- A nitrogen atmosphere focuses the heat flux transferred to the anode in a narrower area than the argon arc that spreads the heat flux to the anode in a wider area but heat fluxes are greater in the nitrogen arc than in the argon arc.

- It was found that an argon atmosphere presents higher electric conductivities, which results in the larger arc size, lower voltages and ohmic resistances than a nitrogen arc for the same operating conditions of the welding process.

\section{ACKNOWLEDGEMENTS}

Authors would like to thank DGAPA-PAPIIT of the National Autonomous University of Mexico for financial support through the project number IN115617, entitled: "Determinación de las características magnetohidrodinámicas de plasmas de soldadura mediante simulación numérica y teorías de escalamiento".

\section{REFERENCES}

1 Maecker H. Plasmaströmungen in Lichtbögen infolge eigenmagnetischer Kompression. Zeitschrift für Physik. 1955;2:198-216

2 Lowke JJ, and HC Ludwing. A simple model for high-current arcs stabilized by forced convection. Journal of applied physics. 1975;8:52-60.

3 Squire HB. The round laminar jet. Quart. Journal Mech. and Applied Math. 1951;3:321329.

4 Ramakrishnan S, and AD Stokes. An approximate model for high currents free-burning arcs. J. Phys. D: Appl. Phys. 1978;11:2267-2280.

5 Ramakrishnan S, and B Nuon. Prediction of properties of free burning welding arc columns. J. Phys. 1980;13:1845-1853.

6 Allum CJ, Gas flow in the column of a TIG welding arc. J. Phys. D: Appl. Phys. 1981;14:1041-1059.

7 Hsu KC, K Etemadi, and E Pfender. Study of the free-burning high-intensity argon arc. J. Appl. Phys. 1982;3:1293-1302.

8 McKelliget $\mathrm{J}$, and $\mathrm{J}$ Szekely. Heat transfer and fluid flow in the welding arc. Metallurgical Transactions A. 1986;17:1139-1148. 
9 Choo RTC. Mathematical modeling of heat and fluid flow phenomena in a mutually coupled welding arc and weld pool [thesis]. Cambridge: Massachusetts Institute of Technology; 1990.

$10 \mathrm{Kim} \mathrm{WH}$, HG Fan, and SJ Na. A mathematical model of gas tungsten arc welding considering the cathode and the free surface of the weld pool. Metallurgical and Materials Transactions B. 1997;28B.

11 Nestor $\mathrm{OH}$, Heat intensity and current density distributions at the anode of high current, inert gas arcs. Journal of applied physics. 1961;5:1638-1648.

12 HG Fan, and R Kovacevic. A unified model of transport phenomena in gas metal arc welding including electrode, arc plasma and molten pool. J. Phys. D: Appl. Phys. 2004;37:2531-2544.

13 M Tanaka, and JJ Lowke. Predictions of weld pool profiles using plasma physics. J. Phys. D: Appl. Phys. 2007;40:R1-R23.

14 Lee YS, and SJ Na. A numerical analysis of a stationary gas tungsten welding arc considering various electrode angles. Supplement of the welding journal, 1996:269-279.

15 Goodarzi M, R Choo, and JM Toguri. The effect of the cathode tip angle on the GTAW arc and weld pool: I. Mathematical model of the arc. Journal of Physics D-Applied Physics. 1997;5:2744-2256.

16 Lowke JJ, R Morrow, and J Haidar. A simplified unified theory of arcs and their electrodes. J. Phys. D: Appl. Phys., 1997;30:2033-2042.

17 Ushio M, M Tanaka, and SC Wu. Analytical approach to anode boundary layer of gas tungsten arcs. Trans. JWRI. 1996;2:9-21.

18 Haidar J, and JJ Lowke. Effect of $\mathrm{CO} 2$ shielding gas on metal droplet formation in arc welding. IEEE Transactions on Plasma Science. 1997;5:931-936.

19 Boulos MI, Fauchais P, and Pfender E. Thermal Plasmas-Fundamentals and Applications. Vol 1. NewYork: Plenum; 1994.

20 Ramírez MA, G Trapaga, and J McKellight. A comparison between two different numerical formulations of welding arc simulation. Institute of Physics Publishing, UK, 2006:275-296.

21 Patankar SV. Numerical heat transfer and fluid flow Computational Methods in Mechanics and Thermal Sciences. 1st ed. New York: McGraw-Hill; 1980. 